\title{
Intangible Radiation Effect of Electronic-Skin (E-Skin)
}

\author{
Rimmy Chuchra ${ }^{1}$, R. K. Seth ${ }^{2}$, Ramesh Chander ${ }^{3}$, Davinder Rana ${ }^{4} \&$ Sunil Kumar ${ }^{5}$ \\ Department of Computer Science \& Engg ${ }^{1,3,4,5}$ \\ Department of Physics ${ }^{2}$ \\ Sri Sai College of Engg and Technology, Manawala (Amritsar) ${ }^{1,3,4,5}$ Punjab (India) \\ DAV University, Jalandhar ${ }^{2}$
}

\begin{abstract}
Acting like a slow poison for human beings, the E-skin technology potentially harms the cell tissues due to radiation effect. The paper discusses about the increase in number of human deaths as a result of use of this smart technology, which had been developed for the welfare of humans. Currently, the computer systems are widely and continuously being used by humans across the globe and this continuous utilization of technology causes health problems due to its direct linkages with E-skin. In skinput technology, the E- skin is an integral part and is used as aninput medium for measuring the electrical activities in human body. The electronic skin works on the principle similar to human skin wherein sensation of touch is prominent, when this electronic skin is embedded into human body. The authors are of the view that this direct linkages of E-skin is significantly harmful and proposes a methodology that uses wireless antenna as an interface between electronic skin and computer system. In the absence of wireless antenna that would act as an interface, the exchange of data between electronic skin and computer system would not be possible. The computer aided diagnosis based on skinput technology provides a real time health monitoring data for maintaining patient's records on regular basis and to tackle the problem in emergencies more efficiently.
\end{abstract}

\section{Keywords}

Electronic skin, human skin, wireless antenna, Computer system, Human computer Interaction (HCI).

\section{INTRODUCTION}

For providing immediate treatments, doctors make regular contact with their patients through electronic skin and send their suggestions on the basis of real time health monitoring data as early as possible. By utilizing this skinput technology patient's heartbeat rate, glucose level and blood pressure can be easily measured by the doctors.

E-Skin is a communication technology that uses thin electronic material for data monitoring which is mimics to human skin and provides a closer view of electronic interaction [4][5][14] with a person. It is known that human skin naturally supports different modalities like pushing, pressing and sneezing etc.that shows its flexible behavior. During the use of skinput technology, the input can only be applied through skin surface [3]. Once user embed or paste on human body, then it will start diagnosing several different parameters of the body; as an example: blood sugar, heart beat and temperature etc. The shape of electronic skin is just like tattoo as simple as to paste on the body. The main motive to paste electronic skin on human skin is only to record all the activities or to measure all the activities such as pulses, movements, pulse rate, heartbeat rate and blood pressure of human body. The human body immediately creates its own body area network (BAN)[12] as the E-skin comes in contact with the bodyand start reading all the electrical activities occurring in the human body. The main advantage of using this technology is that it does not require additional configuration [12], such as more equipment's or devices for attachment, extra effort and does not require more charges. The complete working is based upon bio-sensors. In the absence of bio-sensors, it cannot read all electrical activities going inside the human body. One distinct feature of biosensors is that it is highly sensitive to temperature and pressure [1] [7] and its main function is provide or record real time health monitoring data [2][8]. In addition, biosensors also have one more unique feature that it provides a special treatment on printed card. As studied in literature by the authors, some of the medicine researchers are called this technology as a wearable skin health monitoring technology [15][19] and some of them are called next generation structural health monitoring computer diagnostic technique [11] [16]. The only purpose to utilize this technology is real time health monitoring that helps to save human life during emergencies and provides a smart treatment method in a short duration of time. Several different types of diseases can be diagnosed using this new technology. There are some certain features of this new designed wearable technology [19] that shows its more popularity in today digital world. Some of most important are listed below:

- $\quad$ Electronic-Skin easily stitched with human skin [9] just like a tattoo[15].

- Use of tactile sensors or bio sensors in polymer electronics creates system intelligence that are embedded in smart applications[10] as an example in smart clothing, soft robotics and wallpaper designing [18].

- $\quad$ Its Ultra-skin work like rechargeable batteries [17].

- Reduces the wire and weight of electronic devices [13].

- It provides two way exchange of information whose main function is to read and send the measured signals to the attached external device that may be any computer system. Such type of harmonization between man and machine converts humans present into CYBROG FUTURE.

- $\quad$ Provides a touch with light only- it gives a new way of machine interfacing.

Electronic skin has one major demerit: when electronic skin is inserted or stitched on human skin then its continuous and longer use may create a variety of skin infections, allergic reactions and some other skin problems that ultimately cause skin cancer leading towards additional health issues. 


\section{USE OF WIRELESS ANTENNA WITH SKINPUT TECHNOLOGY}

In this paper authors proposed a methodology for maintaining real time health monitoring record and also how to reduce risk factors. Wireless antenna may serves as a backbone for this skinput technology. This technology uses wireless antenna as an interface between electronic skin and computer system at remote. In the absence of interface the interaction between electronic skin and computer system is not possible. Through electronic skin computer based diagnostic technology, the immediate treatment is easily available to the patients and the doctor may be in regular contact with the patients. The main advantage of utilization this technology the emergency can be handled easily while doctor is not available on site to save humans life. Utilizing this technology, the researchers are trying to make the technology easily available to the society so that common man gets maximum benefit of the technology. At the end, authors suggested this method can only be used for handling emergencies or sometimes when you need for some urgent treatment not on regular or daily basis. The regular use of skinput technology may harm human body due to radiation effects. Therefore, for reducing the risk factors due to radiations, skinput technology can effectively be used along with antenna as and when required in case of handling emergencies or critical conditions. Further, the engineers need should conduct research to design different configurations such as folded, rolled up using crumped electronics materials that has no radiation effect on human health and would provide more efficient results.

\section{RESEARCH DESIGN}

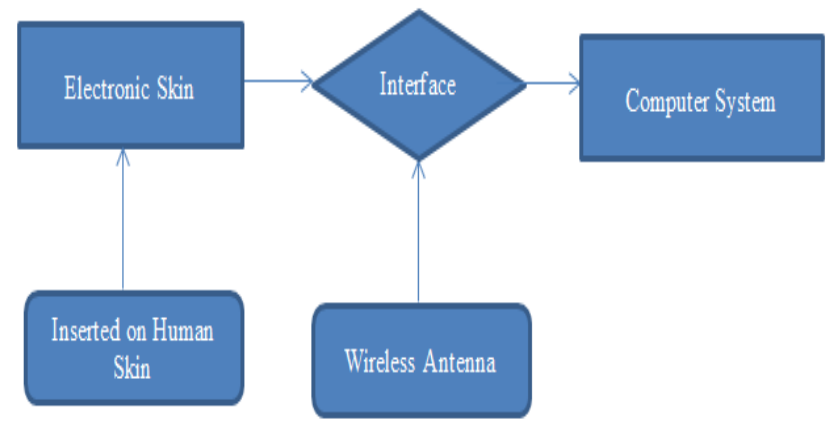

Fig. 1:Interface interaction between electronic skin and computer system.

- The function of wireless antenna is to exchange data from the human body to the computer system. It is inserted on human head whose function is to read all electrical activities going in human body.

- Just take care of one thing the size of antenna should be thin as like human hair.

- The function of electronic skin: - is to provide real time health monitoring data [15][19].

\section{A ROADMAP:-FOR ELECTRONIC- SKIN THAT CAUSE SMART DEATH}
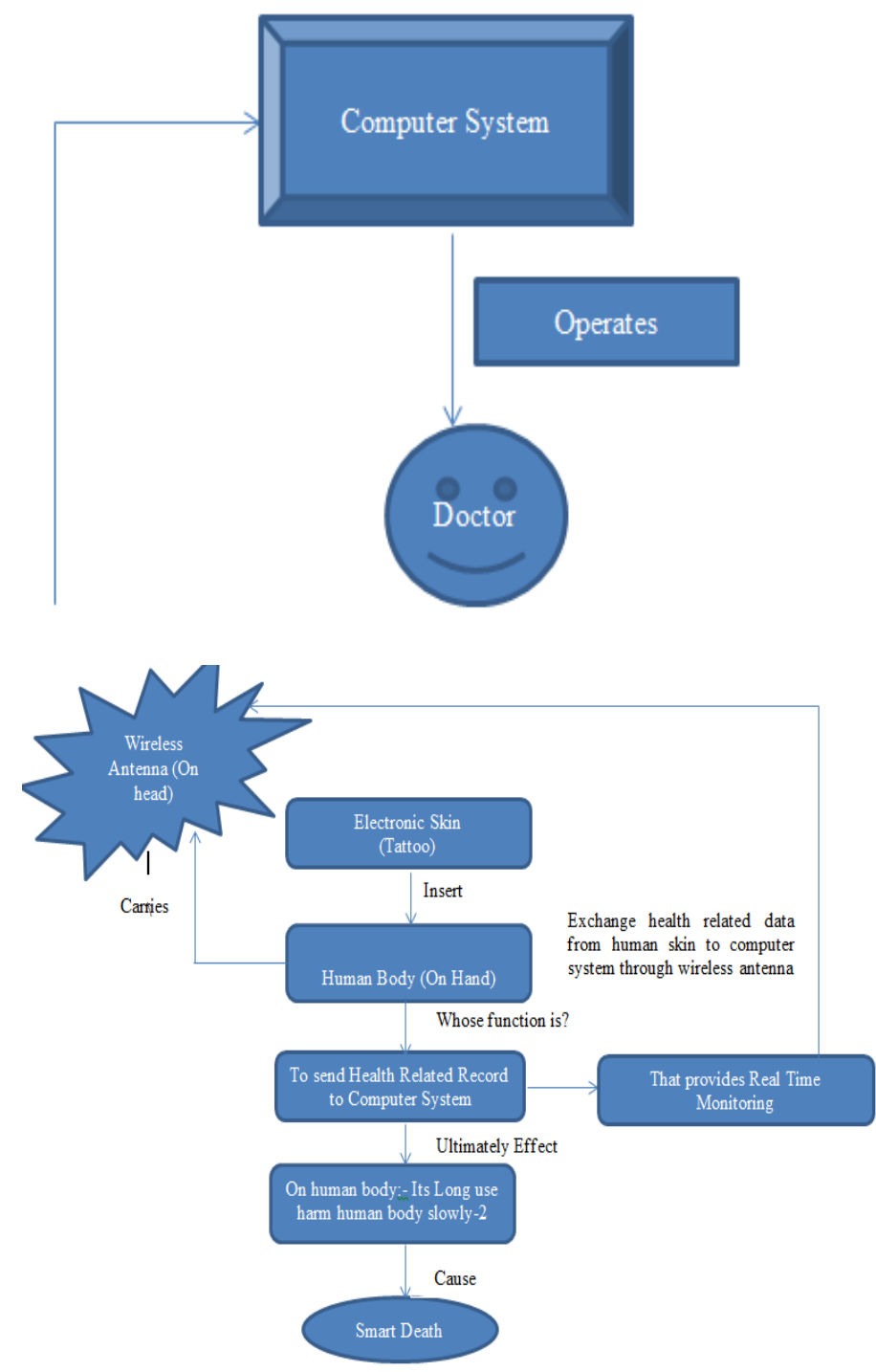

Fig. 2: Doctors interaction with Skinput Technology.

\section{CONCLUSIONS}

The purpose of this paper is to aware the people about skinput technology, where input can only be applied through human skin by pasting electronic skin upon it in the form of tattoo. Using the concept of providing immediate relief to the patients, the doctors can advise or prescribe the medicines to the patients while sitting remotely at the other end using skinput technology embedded with wireless antenna. The radiation effects would be minimal as the diagnostic method using skinput technology does not take much time. The human skin as input provides real time health monitoring data. Once electronic skin pasted on the human skin, the electronic activities occurring inside the body would start recording the activities as it builds its own body area network (BAN).The exchange of data between the human body and the computer system would be possible with the help of wireless antenna as an interface. It is reviewed that this technology is very efficient in emergency cases. 


\section{FUTURE SCOPE}

The researchers could also invent methodology based upon skinput technology to mitigate the radiation effects. The new future technologies could be anti-allergic to human skin so that its longer use would not cause adverse effects on human health and users could use this technology frequently. The materials and integrands to be used would be an important factor for its future utilization.

\section{REFERENCES}

[1] Zhenan Bao,B.H. Tak,Won-gyu bae,2015.A Chameleoninspired stretchable eskin with interactive color changing controlled by tactile sensing, Nature communications.

[2] Gajanan K.Kharte, Archana \& Ghotkar, March 2014.Study of vision based hand gesture recognition using Indian sign language, International journal of smart sensing and intelligent systems.

[3] Jurgoan Steimle and VikramMehtaMay 2014.More than touch: Understanding how people use skin as an input surface for mobile computing, ACM, Canada.

[4] Hartenstein, M.J, Holmes.R, Keltner.D, 2009.In the communication of emotion via touch,in emotion-09, American Psychological Association.

[5] Hertenstein M.J \&Keltner.D App \& Jaskolta A.R, 2009. Touch Communicates Distinct Emotions-In emotions-06, American Psychological Association.

[6] Vladimir J.Lumelsky, June 2001.Sensitive Skin,IEEE Sensors Journals.

[7] K.R Nataraj, K.R Rekha,January 2014.Human hand recognition system based on moving object detectioncolor of the skin and face recognition system, International journal of advanced research in electrical and electronics \& instrumentation engineering.

[8] John Vilson \& Burack Karaguzel,April 2006.Sensors on textile substrates for home-based health care monitoring, Proceeding of the first Distributed diagnosis and home health care conference, IEEE, Virginia.
[9] M.Sravanthy \& K.Ranganath, 2007.ESKIN the sense of touch, International Journal of computer science engineering and technology.

[10] Mark Love and Julian Lina, 2002. A Large Area force sensor for smart skin applications, USA.

[11] Narito Kurata \& Manuel E.Ruiz-Sandoval, Smart Sensing Technology-opportunities \& challenges, TokyoJapan.

[12] David Kotz, Ryan Halter \& Joseph Skinner, 2014. A Wearable systems that knows who wears it, ACM, USA.

[13] Oliver G.Schmidt, Takao Someya \& Tsuyoshi Sekitani, January 2014.Imperceptible Magnet electronics, Nature Communications.

[14] Michael Storper and Chirag Rabari, January 2013. The digital skin of cities.

[15] Sukanya Jadhav,Renia Doge \& Manoj Thakur, December 2014. Flexible eskin,INPRESSCO (International Press Conference).

[16] Adel Ali,A.I Jumaily \& Ammara Masood, 2013.Computer Aided Diagnostic support system for skin cancer: A Review of techniques and algorithms, International journal of biomedical imaging, Hindwai Publishing Corporation.

[17] A.L.N Stevels \& A.J Jansen, Human Power- A sustainable Option for electronics design for sustainability,IEEE Program,Netherlands.

[18] J.Vanfleteren, T.Vervust\& R.Verplanke, October 2015.Stretchable engineering technologies for the development of advanced stretchable polymeric systems, IEEE Conference Paper.

[19] Kristof Van Laerhoven, Masaaki Fukumotto, Daniel Gatica and Daniel Roggen, 2014.Wearables are here to stay, IEEE, 2014 